Leben in der Krise 


\section{This page intentionally left blank}




\section{Martin Lindner \\ Leben in der Krise \\ Zeitromane der Neuen Sachlichkeit und die intellektuelle Mentalität der klassischen Moderne}

Mit einer exemplarischen Analyse des Romanwerks von Arnolt Bronnen, Ernst Glaeser, Ernst von Salomon und Ernst Erich Noth

Verlag J. B. Metzler Stuttgart · Weimar 
Die Deutsche Bibliothek - CIP-Einheitaufnahme folgt

\section{Lindner, Martin:}

Leben in der Krise : Zeitromane der neuen Sachlichkeit und die intellektuellen Mentalität der klassischen Moderne ; mit einer exemplarischen Analyse des Romanwerks von Arnolt Bronnen, Ernst Glaiser, Ernst von Salomon und Ernst Erich Noth /

Martin Lindner. - Stuttgart ; Weimar : Metzler, 1994

(Metzler-Studienausgabe)

ISBN 978-3-476-00996-8

ISBN 978-3-476-00996-8

ISBN 978-3-476-03530-1 (eBook)

DOI 10.1007/978-3-476-03530-1

Dieses Werk einschließlich aller seiner Teile ist urheberrechtlich geschützt. Jede Verwertung außerhalb der engen Grenzen des Urheberrechtsgesetzes ist ohne Zustimmung des Verlages unzulässig und strafbar. Das gilt insbesondere für Vervielfältigungen, Übersetzungen, Mikroverfilmungen und die Einspeicherung und Verarbeitung in elektronischen Systemen.

(C) 1994 Springer-Verlag GmbH Deutschland

Ursprünglich erschienen bei J. B. Metzlersche Verlagsbuchhandlung und Carl Ernst Poeschel Verlag GmbH in Stuttgart 1994 


\section{Inhaltsverzeichnis}

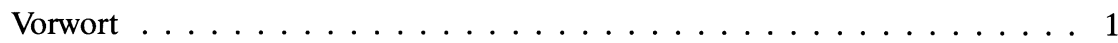

\section{Teil 1}

\section{Lebensideologie}

0 . Eine erste Definition der Lebensideologie $\ldots \ldots \ldots \ldots$. . . . . 5

1. Die Krise als Zentrum lebensideologischen Denkens:

Tektonische Spannung zwischen Lebensstrom und Lebensform $\ldots \ldots \ldots$

2. Lebensideologische Sprach- und Erkenntniskritik . . . . . . . . . . 11

3. Lebensideologische Metaphysik: Die immanente Transzendenz des Lebens . 15

4. Leben vs. Geist: Radikale und gemäßigte Lebensideologie . . . . . . . . . 19

5. Lebensideologische Psychologie: Das Unbewußte und seine Rolle im Zyklus von Krise, Tod und Wiedergeburt . . . . . . . . . . . . . . . 24

6. Lebensideologische Soziologie: Die Masse und die Mitte . . . . . . . . . 40

7. Lebensideologische Geschichtsphilosophie:

Der Strom der Zeit bricht die Dämme . . . . . . . . . . . . . . . . . 53

8. Mythos vs. Utopie: Konservative und progressive Geschichtsentwürfe . . . . 60

9. Die geschichtliche Rolle der Eliten . . . . . . . . . . . . . . . . 65

10. Lebensideologische Geographie und Geopolitik: Lebensraum . . . . . 73

11. Lebensideologische Biologie: Neovitalismus, Darwinismus, Rassismus . . . 80

12. Die neue Polarität der Geschlechter . . . . . . . . . . . . . . 84

13. Querverbindungen zu anderen Diskursen:

Lebensideologische Ökonomie, lebensideologische Physik . . . . . . . 87

14. Die Funktion der Literatur als lebensideologischer Metadiskurs . . . . . . .

15. Die Denkfiguren des Heroischen Realismus:

Schicksal, Bindung, Mythos, Opfertod . . . . . . . . . . . . . . 93

16. Erkenntnistheoretische Denkfiguren der Lebensideologie:

Ganzheit, Polarität, Typus, Tat . . . . . . . . . . . . . . 102

17. Zwei lebensideologische Schlüsselmetaphern: Wurzel und Kristall . . . . . 113 


\section{Teil 2 (Exkurs) \\ Zur Entstehung und Entwicklung der Lebensideologie im historischen Kontext}

1. Vitalismus, Lebensphilosophie, Lebensideologie . . . . . . . . . . . . . 119

2. Die Krise des Bildungsbürgertums um 1900 . . . . . . . . . . . . . . . . 122

3. Voraussetzungen: Varianten wilhelminischer Kulturkritik . . . . . . . . 127

4. Neuromantik und neuklassische Sachlichkeit . . . . . . . . . . . . . . 131

5. Die lebensideologische Transzendentalpolitik: Tatgesinnung . . . . . . . . 137

6. Die fünf Phasen der lebensideologischen Epoche $(1890$ - 1955) . . . . . . . 142

Teil 3

Neue Sachlichkeit

1. Die geistige Situation um 1930: Stereotypen der Zeitkritik . . . . . . . . . 146

2. Die neue Sachlichkeit als lebensideologische Teilströmung:

Das Pathos von Krise, Kritik und Katharsis . . . . . . . . . . . . . . 155

3. Die Struktur der neusachlichen Psyche: Feuer und Eis . . . . . . . . . . 168

4. Die Geschichtsphilosophie der Neuen Sachlichkeit:

Umschlag in das kristalline Kollektiv . . . . . . . . . . . . . . . . . . 175

5. Neusachlicher Irrationalismus und Innere Emigration:

Wovon man nicht sprechen kann, darüber muß man schweigen . . . . . . . . 183

6. Die Rolle des neusachlichen Intellektuellen in der Massenzivilisation . . . . 195

\section{Teil 4}

Lebensideologische Strukturen im neusachlichen Zeitroman (Arnolt Bronnen, Ernst Glaeser, Ernst von Salomon, Ernst Erich Noth)

1. Vier unterschiedliche Konzeptionen von Leben . . . . . . . . . . . . . . 207

2. Die Sehnsucht verläuft von Nord nach Süd, der Wille von West nach Ost:

Der europäische Lebensraum und die deutsche Mitte . . . . . . . . . . . . 239

3. Der soziale Lebensraum und die Problematik der Mittelschichten . . . . . . 249

4. Der Zerfall der bürgerlichen Gesellschaft und die Entstehung neuartiger Kollektive . . . . . . . . . . . . . . . . . . . 266

5. Kritik oder Dynamit: Die Mission der neusachlichen Protagonisten . . . . 276

6. Provisorisch leben: Das Pathos der Existenz . . . . . . . . . . . . . . . 293

7. Die bedrohliche Faszination der Sexualität und der Frauen . . . . . . . . 320

8. Die reißende Flut der Geschichte: Fatalismus und utopische Vision . . . . . 331

9. Der Nationalsozialismus als Lebens-Katastrophe . . . . . . . . . . . . . 353 
10. Die Funktion des neusachlichen Erzähltextes:

Wünschenswert ist die Anfertigung von Dokumenten . . . . . . . . . . . . . . 361

11. Epilog: Die neusachliche Generation nach 1945 . . . . . . . . . . . . 372

\section{Teil 5 \\ Anhang}

1. Zur Methodik . . . . . . . . . . . . . . . . . . 377

2. Zur Sekundärliteratur . . . . . . . . . . . . . . . . . . . 383

3. Zu den Textausgaben . . . . . . . . . . . . . . . . 388

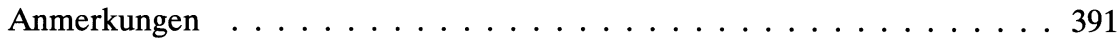

Literaturverzeichnis . . . . . . . . . . . . . . . . . 403 


\section{Vorwort}

Die Feststellung Wolfdietrich Raschs, daß in Deutschland um 1900 der Terminus »Leben« zum »zentralen Begriff der Epoche« wird, ist inzwischen ein kulturgeschichtlicher Gemeinplatz. Wer sich mit Literatur und Denken der neuromantischen oder der expressionistischen Generation befaßt, tut sich schwer, den inflationären Gebrauch des Begriffs »Leben« bzw. seines semantischen Umfelds zu übersehen. Vermutlich ist gerade diese Offensichtlichkeit dafür verantwortlich, daß es die literaturwissenschaftliche Forschung bisher weitgehend für überflüssig hielt, näher zu untersuchen, was es über den einzelnen Text hinaus mit dem semantischen Komplex des »Lebens« im einzelnen auf sich hat. Man begnügt sich gemeinhin mit dem Hinweis auf die Lebensphilosophie, und das bedeutet dann im beiläufigen literaturwissenschaftlichen Sprachgebrauch ungefähr so viel wie: »irgendwie irrational«, »irgendwie dynamisch«, »hat etwas mit Nietzsche, Bergson, Simmel und Dilthey zu tun«.

Damit ist aber, genau besehen, nicht viel gewonnen: »Die« Lebensphilosophie gibt es schon deshalb nicht, weil die genannten Schlüsselfiguren sich weniger als systematische Philosophen denn als Essayisten verstanden. Und das hatte einen guten Grund: Die in der Umbruchszeit der Jahrhundertwende so dringend ersehnte »lebendige « Philosophie konnte, so glaubte man, nicht an akademischen Lehrstühlen entstehen, wo es nur »tote « Systeme gebe, bestenfalls die erstarrten Sedimente ehemals vitalen Denkens. Das eigentliche Medium, in dem die Lebensphilosophie konzipiert, durchgesetzt und weiterentwickelt wurde, war die Literatur: der literarische Essay in der Nachfolge Nietzsches, die Lyrik Georges, Hoffmansthals und Rilkes, der philosophische Roman Thomas Manns, Hesses und Wassermanns, die Dramatik der Neuromantik und mehr noch des Expressionismus, nicht zu vergessen die triviale Weiterverwertung und massenhafte Verbreitung vitalistischer Klischees in der völkischen Literatur von Kolbenheyer, Löns, Flex, Grimm u.v.a.

Diese Untersuchung stellt nun einige Hypothesen auf und versucht, sie zumindest plausibel zu machen:

Erstens handelt es sich bei dem semantischen Komplex »Leben «/»Krise«/»Wiedergeburt « um eine komplexe Denkstruktur, die trotz ihrer programmatischen Vagheit präzise identifiziert und analysiert werden kann. Diese Denkstruktur, die in ihrer ganzen Reichweite den Zeitgenossen nur zum Teil bewußt war, nenne ich im folgenden »Lebensideologie «(LI). Den ehrgeizigsten und erhellendsten Versuch ihrer Beschreibung hat im übrigen Robert Musil, selbst Angehöriger der Epoche, in 
seinem Roman »Der Mann ohne Eigenschaften« unternommen. Die LI ist kein philosophisches System. Sie läßt sich am ehesten als ein in sich zusammenhängender Ausschnitt »intellektueller Mentalität« kennzeichnen - jedenfalls weist sie die Merkmale auf, durch die dem Historiker Peter Burke zufolge der Gegenstand der Geschichte intellektueller Mentalitäten definiert ist: "Zum ersten durch ihre Betonung der kollektiven anstelle der individuellen Einstellungen. Zweitens durch den Nachdruck, den sie auf unausgesprochene und unbewußte Annahmen legt [...] Drittens schließlich durch ihr Interesse für die Struktur von Meinungen, für Kategorien, für Metaphern und Symbole, dafür wie die Leute denken und nicht nur dafür, was sie denken.« [1]

Zweitens bestimmt die Denkstruktur der LI, in ihren gleichzeitigen weltanschaulichen Variationen und aufeinander aufbauenden Entwicklungsstufen, ganz entscheidend die Literatur (und das Denken der bürgerlichen Intelligenz überhaupt) von etwa 1890 bis einschließlich der existenzphilosophisch geprägten Strömungen der 50er Jahre (z.B. dem frühen Böll und dem frühen Andersch). Eine solche Epoche ließe sich in einem Schlagwort wohl am ehesten als deutsche »klassische Moderne« bezeichnen. Es ergäbe sich damit ein Zusammenhang, der einerseits abzugrenzen ist gegen die vornaturalistische Literatur und andererseits gegen die Literatur der jüngeren Gruppe 47 (etwa Enzensberger, Walser, Höllerer). Für die DDR-Literatur dürfte im übrigen ein ähnlicher Schnitt gelten: Becher und Huchel etwa bewegen sich noch im Kontext der lebensideologischen Denksstruktur, Christa Wolf nicht mehr. Das heißt natürlich nicht, daß in der Literatur seit 1955/1960 keine Einflüsse lebensideologischen, etwa existentialistischen und vitalistischen, Denkens mehr nachweisbar wären: Ein älterer Autor wie Ernst Jünger hat seine lebensideologischen Denkfiguren unbeindruckt beibehalten und gerade eben greift ein (relativ) jüngerer Autor wie Heiner Müller massiv vitalistische Denkmuster auf. Aber das ideologische Gravitationszentrum ist der Kultur verlorengegangen, der Schlüssel, der einzelnen derartigen Äußerungen bis in die 50er Jahre hinein einen tieferen $\mathrm{Zu}$ sammenhang und Sinn gegeben hatte. Die kontextlosen Brocken der Lebensideologie können seitdem als Bausteine in neuen Strukturen verwendet werden, die im übrigen erst noch zu analysieren wären.

Drittens demonstriert diese Untersuchung, daß gerade den nur scheinbar so klaren und wenig komplexen Texten der Neuen Sachlichkeit, die der Literaturwissenschaft bisher kaum einmal eine tiefgehende Analyse wert waren, eine zentrale Funktion im Zusammenhang der lebensideologischen Epoche zukommt. In den 20er Jahren war das lebensideologische Denksystem durchgesetzt und selbstverständlich geworden. Die Wende zur angeblich ideologiefreien »Sachlichkeit« lag - trotz der rhethorischen Betonung eines radikalen Bruchs mit der Vergangenheit - selbst in der spezifischen Binnenlogik lebensideologischen Denkens begründet. In der Literatur wurden nun die bis dahin überdeutlichen lebensideologischen Denkfiguren nun zu lakonischen Chiffren verknappt, deren Entschlüsselung beim Leser die bewußte oder intuitive Kenntnis der lebensideologischen Zusammenhänge voraussetzte. Zugleich entwickelte sich eine fatalistische Spielart lebensideologischen Denkens, de- 
ren Wirksamkeit dann von der Inneren Emigration bis in die 50er Jahre reichte. Erst dann kam die Dynamik lebensideologischen Denkens zum Stillstand, das Denksystem wurde durch andere Systeme überlagert und löste sich allmählich auf.

Viertens wird an detaillierten Textanalysen von neusachlichen »Zeitromanen « [2] im einzelnen gezeigt, was die konkrete Wirklichkeit des lebensideologischen "Denksystems« ausmacht, das ja eigentlich nur als Abstraktum existiert, quasi als Essenz zumindest der schriftlichen Äußerungen der Epoche. Gegenstand der exemplarischen Analysen sind die zwischen 1927 und 1937 erschienenen Zeitromane zweier »linker« und zweier »rechter « Autoren (Ernst Glaeser und Ernst Erich Noth bzw. Arnolt Bronnen und Ernst von Salomon). Es wird gezeigt, wie die lebensideologische Denkstruktur das Weltbild einzelner Individuen und die literarische Struktur ihrer Werke bestimmt, welche individuellen und originellen Standpunkte dem einzelnen möglich waren und wie sich solche Standpunkte Schritt für Schritt verändern. Dabei ergeben sich u.a. wichtige Erkenntnisse über die gemeinsamen Denkfiguren und spezifischen Unterschiede zwischen »linken « und »rechten « Intellektuellen, die Einschätzung des Nationalsozialismus durch die junge bürgerliche Intelligenz und v.a. auch über bis heute nachwirkende Konzepte, die die Dynamik der modernen Industrie- und Massengesellschaft verarbeiten sollten.

Damit hoffe ich schließlich fünftens, ein mögliches Modell vorzuführen, das es erlaubt, auch für andere Epochen Literatur und Kulturgeschichte in Beziehung zu setzen, ohne auf der einen Seite die Eigendynamik des literarischen Diskurses zu unterschlagen und ohne auf der anderen Seite eine schöngeistige Isolierung der Literaturwissenschaft zu fördern. Es sollte deutlich werden, welche Bedeutung Literatur (und damit auch die Literaturwissenschaft) für das Selbstverständnis wie für das nachträgliche Verstehen einer Kultur haben kann: in jedem Fall als privilegierte Quelle, die es ermöglicht, sehr subtile Symptome kulturellen Wandels wahrzunehmen, und - jedenfalls in der Epoche 1890 - 1955 - zugleich als Medium, das die Welt nicht nur interpretieren, sondern auch verändern kann. 